\title{
A Robust and Accurate Traveltime Calculation from Frequency-domain Two-way Wave-equation Modeling Algorithm
}

\author{
Yilong Qin ${ }^{1 *}$, Seungwon Ko ${ }^{2}$, Changsoo Shin $^{2}$, Zhongjie Zhang ${ }^{1}$, \\ Youngtak $\mathrm{Seo}^{2}$, Uk $\mathrm{Han}^{3}$ and Yun Chen ${ }^{1}$ \\ ${ }^{1}$ Institute of Geology and Geophysics, Chinese Academy of Sciences, Qijiahuozi, Beijing 100029, China \\ ${ }^{2}$ Seoul National University, School of Civil, Urban and Geosystem Engineering, Seoul, 151-742, Korea \\ ${ }^{3}$ Department of Environment Science, Korea Military Academy, Seoul, 139-799, Korea.
}

\begin{abstract}
We improve the accuracy and stability of traveltime calculation method using frequency-domain modeling algorithm. We perform a parameter analysis to obtain the optimum combination of frequency and damping factor and thus improve the accuracy of traveltime. Then we obtain the empirical formula for our numerical algorithm. Lastly, we propose the adaptive frequency and the adaptive damping factor for an inhomogeneous model to eliminate the distortion in the traveltime contour. Twodimensional numerical examples verify that the proposed algorithm gives a much smaller traveltime error and a better traveltime contour for the complex model. Compared to the other two methods, this algorithm computes traveltime that is close to a directly transmitted wave. We demonstrated our algorithm on 2D IFP Marmousi models, and the numerical results show that our algorithm is a faster traveltime calculation method of a directly transmitted wave for imaging the subsurface and transmission tomography.
\end{abstract}

Key words : traveltime calculation, frequency-domain modeling, parameter analysis

\section{INTRODUCTION}

A robust and accurate traveltime calculation is of critical importance for transmission tomography, refraction tomography, earthquake seismology, and prestack Kirchhoff migration. To this end, geophyicists have developed a variety of traveltime calculation techniques. Most of the techniques can be divided into four categories: (1) ray-

Received June 15, 2004; Accepted September 17, 2004

Published October 31, 2004

*Corresponding Author: Yilong Qin

E-mail: yilongqin@mail.igcas.ac.cn

Address: Institute of Geology \& Geophysics Chinese Academy Sciences Qijiahuozi, Chaoyang District, Beijing, China, 100029 tracing method; (2) Eikonal solver (Kim, 2002; Mo and Harris, 2002; Afnimar and Koketsu, 2000; Alkhalifah and Fomel, 2001) (3) Shortest-path algorithm (Cheng and House, 1996; Cao and Greenhalgh, 1993; Fischer and Lees, 1993); and (4) traveltime calculation from a wave equation modeling algorithm (Shin et al., 2002, 2003a, 2003b). These methods have their own characteristics. Compared with other methods, the traveltime calculation using the wave-equation modeling algorithm has its own advantages. Its (1) more suitable for a computation of multi-shot traveltime used in Kirchhoff migration; (2) can be modified to calculate the most-energetic traveltime (Shin et al., 2003a, 2003b; Nichols, 1996); (3) can be easily extended to elastic media or anisotropic media; and (4) can simultaneously calculate traveltime and amplitude.

The traveltime calculation using the frequency-domain modeling algorithm was originally proposed by Shin et $a l$. (2003a). They applied a complex frequency (the real part is the frequency, and the imaginary part is the damping factor) into the frequency-domain wave-equation and then approximated the damped wavefield in the depth by using the Dirac delta function. Thus, they transformed the difficult traveltime picking problems into an easier maximum-arrival picking problem. For this algorithm, the choice of frequency and damping factor is critical for accuracy of the traveltime since different combinations of the frequency and the damping factor give different traveltimes. Conventionally, the frequency and the damping factor are determined from dispersion analysis (Shin et al., 2003a). However, the dispersion analysis has difficulty in giving the optimum combination of frequency and damping factor since a different dispersion error limit derives a different frequency and damping factor. Essentially, the dispersion analysis only considers the dispersion error and neglects the assumption of high-frequency approximation. Actually, for the traveltime calculation from the frequency-domain modeling algorithm, accurate traveltime depends on not only dispersion error but also the high-frequency approximation. Only a very small grid size can satisfy both of the requirements. Unfortunately, because of the limitations of computer memory and speed, the number of model grids cannot be too large. When the grid size is fixed, there is a trade-off between the disper- 
sion and assumption of high-frequency approximation, that is, the small dispersion requires a low frequency, but the high-frequency approximation requires a high frequency. Therefore, to obtain the best traveltime, we must find the optimum combination of the frequency and the damping factor. In addition, the traveltime contour computed by the basic algorithm is distorted for heterogeneous media.

In this paper, we improve the accuracy and stability of the traveltime calculation from the frequency-domain waveequation modeling algorithm. The accuracy is improved by using parameter analysis to obtain the optimum combination of the frequency and the damping factor. The stability is improved by using an adaptive frequency and an adaptive damping factor to eliminate the distorted traveltime contour in an inhomogeneous medium.

We begin by reviewing the numerical solution of the frequency-domain two-way wave equation. We review the simple technique that computes the traveltime. Next, we propose the parameter analysis to obtain the optimum combination of the frequency and the damping factor and the adaptive complex frequency to eliminate the distorted traveltime contour. Finally, we give the numerical examples for the IFP Marmousi model and compare our traveltime with the most-energetic and Straight-Ray-Technique (SRT) traveltime. We also generate Kirchhoff-migrated images by using our adaptive traveltime for the $2 \mathrm{D}$ Marmousi model.

\section{BASIC THEORY}

\section{Damping factor}

We assumed that a seismic signal observed at a receiver in depth can be approximated by a series of weighted spikes (Fig. 1a). The weighted series of spike can be expressed as

$$
u(t)=\sum_{n} A_{n} \delta\left(t-t_{n}\right)
$$

where the $A_{n}$ and $t_{n}$ are the amplitude and the $n$th digitized time (countered from the first-arrival event), respectively. In general, if we multiply equation (1) by a strong damping factor, we can suppress all the events following the first arrival, as shown in Fig. 1b, and thus approximate the solution as

$$
u^{*}(t)=u(t) e^{-\alpha t} \cong A_{1} e^{-\alpha t} \delta\left(t-t_{1}\right)
$$

where $A_{1}$ and $t_{1}$ are the amplitude and traveltime of the first arrival. By suppressing all the wave events following the first arrival, we transform the difficult traveltime picking problems into an easier maximum-arrival picking problem.

\section{Two-way frequency-domain modeling}

We calculate traveltime by using a two-way frequency-
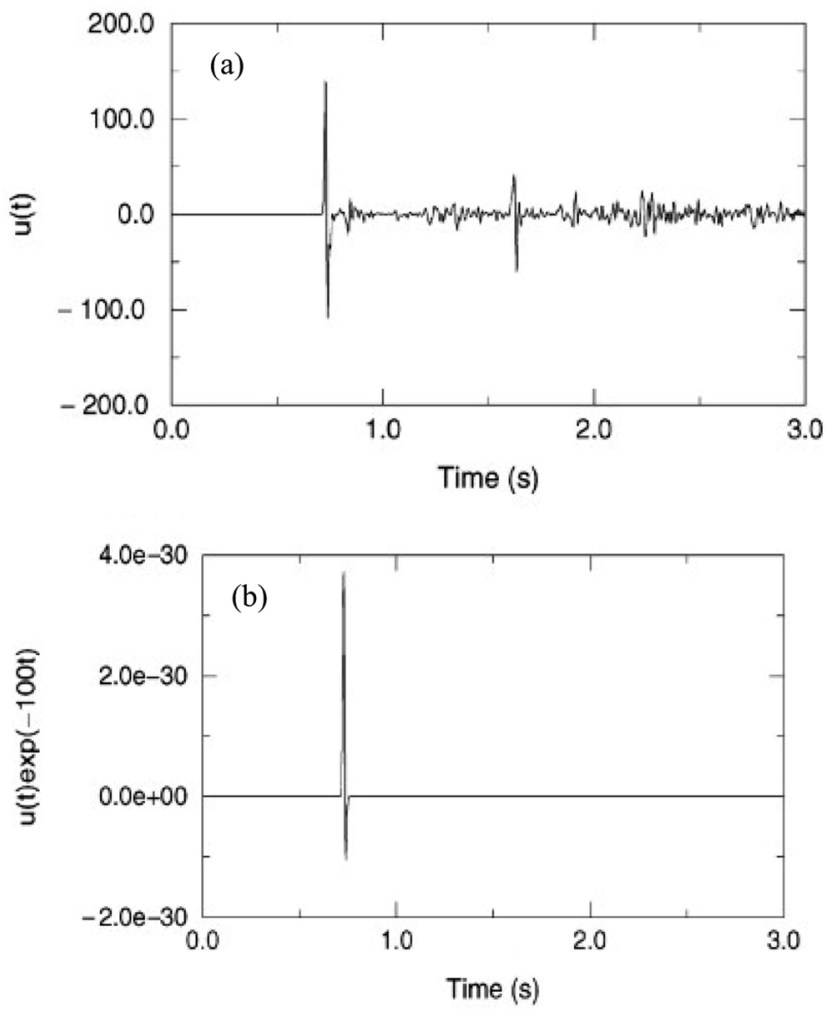

Fig. 1. Synthetic seismograms for a 2-D earth model. (a) the seismic signal can be approximated by a series of weighted, and (b) delta-like wavefield obtained by introducing a strong damping factor $\alpha=100$

domain wave equation. The scalar wave equation for a homogeneous isotropic medium in the frequency domain is

$$
\nabla^{2} u+\frac{\omega^{2}}{v^{2}} u=0
$$

where the $u$ is the pressure field, $\omega$ is the angular frequency, and $v$ is the velocity. The frequency-domain, finite-difference formulation for the scalar wave equation can be written as

$$
\left[\mathbf{K}(\mathbf{m})+i \omega \mathbf{C}(\mathbf{m})-\omega^{2} \mathbf{M}(\mathbf{m})\right] \mathbf{u}(\mathbf{m}, \omega)=\mathbf{f}(\omega)
$$

where $\mathbf{M}, \mathbf{C}$, and $\mathbf{K}$ are the $\mathrm{n} \times \mathbf{n}$ ( $n$ is the number of nodal points) mass, damping, and stiffness matrices, respectively; f and $\mathbf{u}$ are the $\mathrm{n} \times \mathrm{n}$ source and data vectors, respectively; $\mathbf{m}$ is the model vector comprised of the impedance and velocity at each nodal coordinate, and $i=\sqrt{-1}$. For simplicity, we express the above equation as

$$
\mathbf{S}(\mathbf{m}, \omega) \mathbf{u}(\mathbf{m}, \omega)=\mathbf{f}(\omega)
$$

where the complex impedance matrix $\mathbf{S}$ is given by

$$
\mathbf{S}=\mathbf{K}+i \omega \mathbf{C}-\omega^{2} \mathbf{M}
$$


In principle, we could calculate the inverse of $\mathbf{S}$ to obtain the wavefield $\mathbf{u}=\mathbf{S}^{-1} \mathbf{f} \equiv \mathbf{G f}$, where we recognize $\mathbf{G}$ to be the Green function. In practice, we do not explicitly calculate but rather decompose $\mathrm{S}$ into the product of a lower triangular matrix and an upper triangular matrix. We then calculate the wavefield $\mathbf{u}$ by forward and backward substitution. Matrices associated with 2-D finite-difference meshes are amenable to the modern sparse matrix technique.

\section{Traveltime calculation from the frequency-domain wavefield}

We calculate the traveltime from the wavefield, obtained by inserting a complex angular frequency into the frequencydomain wave equation, in the forward modeling. A complex angular frequency is expressed as

$$
\omega^{*}=\omega+i \omega
$$

where $\omega$ is the real angular frequency and $\alpha$ is the suppression factor that is commonly used to prevent wraparound effects inherent in frequency-domain solutions. By virtue of the shifting theorem of Fourier-domain transforms, the factor $\alpha$ suppresses the time-domain solution by $e^{-\alpha t}$. In conventional frequency-domain modeling, we synthesized our time-domain solutions from the Fourier component $\omega^{*}$, where $\alpha$ is a constant and $\omega$ varies, and multiplied the final time-domain results by the inverse of the damping factor, $e^{\alpha t}$.

By using complex frequencies, we can reduce the general multiple event response to a single event. The resulting single event can correspond to the first arrival. According to equations (1) and (2), the time-domain wavefield obtained by the two-way wave-equation with complex angular frequency can be approximated as

$$
u(x, z, t)=\tilde{A}(x, z, t) \delta(t-\tau(x, z, t))
$$

where $\tilde{A}(x, z)=A(x, z) e^{-\alpha t}, u$ is the wavefield, $\tau(x, z, t)$ is the traveltime from the source to a depth point in the subsurface, $A(x, z, t)$ is the amplitude at the depth point in the subsurface, and $\delta$ is the Dirac delta function. In the frequency-domain, the equation above can be written as

$$
u(x, z, \omega)=A(x, z) e^{-i \omega \tau(x, z)}
$$

Differentiating the above equation with respect to gives

$$
\begin{aligned}
\frac{d u(x, z, \omega)}{d \omega} & \approx-i \tau(x, z) A(x, z) e^{-i \omega \tau(x, z)} \\
& =-i \tau(x, z) u(x, z, \omega)
\end{aligned}
$$

From the equation above, we compute the traveltime by dividing $d u / d \omega$ by $i u$. The derivative of wavefield $u$ with respect to $\omega$ can be calculated using forward and backward substitution. For example, taking the partial derivative of equation (5) with respect to complex frequency $\omega$ yields

$$
\mathbf{S}(\mathbf{m}, \omega) \frac{\partial \mathbf{u}(\mathbf{m}, \omega)}{\partial \omega}+\frac{\partial \mathbf{S}(\mathbf{m}, \omega)}{\partial \omega} \mathbf{u}(\mathbf{m}, \omega)=0
$$

After arranging the equation, we obtain

$$
\mathbf{S} \frac{\partial \mathbf{u}}{\partial \omega}=\mathbf{f}
$$

where the virtual source vector $\mathbf{f}$ is associated with the perturbation of the complex frequency and is given by

$$
\mathbf{f}=-\frac{\partial \mathbf{u}}{\partial \omega} \mathbf{u}=(2 \omega \mathbf{m}-i \mathbf{C}) \mathbf{u}
$$

Equation (12) has the same form as equation (5), where $u$ and $f$ are replaced by $\partial \mathbf{u} / \partial \omega$ and $\mathbf{f}$, respectively. Since the $\mathbf{f}$ in equation (5) is constant, the derivative of $\mathbf{f}$ becomes zero. The vector $\mathbf{f}$ is a new source used to compute the derivative of the wavefield $\partial \mathbf{u} / \partial \omega$. Once we factorize the matrix $\mathbf{S}$ and obtain the wavefield $\mathbf{u}$ in the frequency domain, the computation of the derivative wavefield only requires one more forward and backward substitution.

\section{IMPROVEMENTS ON ACCURACY AND STABILITY}

\section{Improvement on the accuracy of traveltime}

For the traveltime calculation from the frequency-domain modeling algorithm, an accurate traveltime requires a

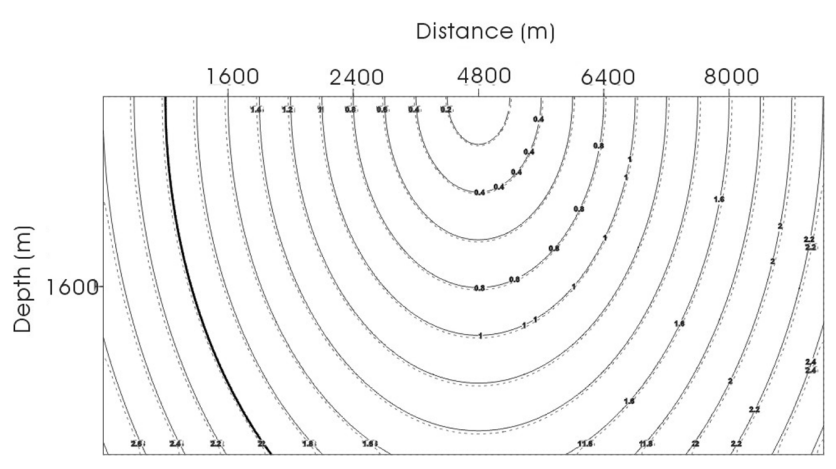

(a)

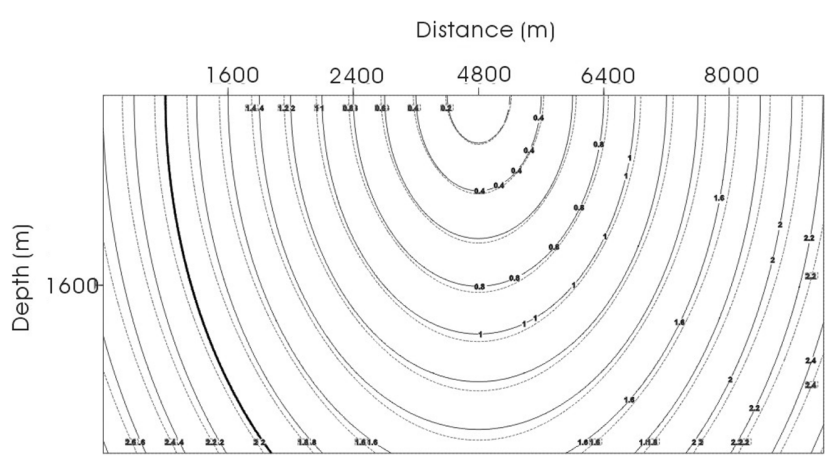

(b)

Fig. 2. Analytic traveltimes (solid lines) and numerical traveltimes (dotted lines) computed by using a different frequency and damping factor (a) $f=1 \mathrm{~Hz}, \alpha=55$; (b) $f=10 \mathrm{~Hz}, \alpha=140$. The velocity is $2.0 \mathrm{~km} / \mathrm{s}$. 
proper selection of the frequency and the damping factor, since different combinations of the frequency and the damping factor give different traveltimes, as shown in Fig. 2. A large frequency can approximate the assumption of high-frequency, but cause large numerical dispersion. Similarly, a large damping factor can strongly damp all the wavefields except the first-arrival event, but require a fine grid to minimize the dispersion error, which consequently increases computational cost. A small damping factor will introduce error in picking the traveltime, because the damped wavefield is approximated by the Dirac delta function. Therefore, in order to obtain an accurate traveltime, we need to consider not only the dispersion error but also the assumption of high-frequency approximation. If we use a very small grid size, we can satisfy both the dispersion and the high-frequency assumption. Unfortunately, for a frequency-domain wave-equation modeling algorithm, the solution of the sparse matrix requires a large memory and a high amount of CPU time. Therefore, we cannot use a very small grid size. For a given grid size, there is a trade-off between the dispersion and the highfrequency assumption. Our problem was how to choose the optimum combination of frequency and damping factor for a given grid size.

Conventionally, the frequency and damping factor are determined from the dispersion analysis (Shin et al., 2003a):

$$
\omega^{*}=\frac{2 \pi V_{\min }}{G \Delta}+i \frac{2 \pi V_{a v g}}{G \Delta}
$$

where $G$ is the number of grid points per wavelength determined from dispersion curves for a given dispersion error limit; $\omega^{*}$ is the optimum complex frequency; $V_{\text {arg }}$ is the average velocity of a given model; $V_{\min }$ is the minimum velocity for one given model, and $\Delta$ is the grid interval.

Since the dispersion analysis determines the optimum complex frequency in terms of only the dispersion error, it is difficult to achieve the optimum complex frequency. Moreover, the dispersion analysis varies with the detailed numerical algorithm. Therefore, we propose the parameter analysis in order to get an optimum combination of frequency and damping factor

To illustrate how we use the parameter analysis to obtain the optimum frequency and damping factor in solving the two-way frequency-domain wave equation by the finitedifference method, we first examine a 2-D homo-geneous half-space model with a $1151 \times 376$ grid set, the grid size of $8 \mathrm{~m}$, and a constant velocity of $2 \mathrm{~km} / \mathrm{s}$. The source is

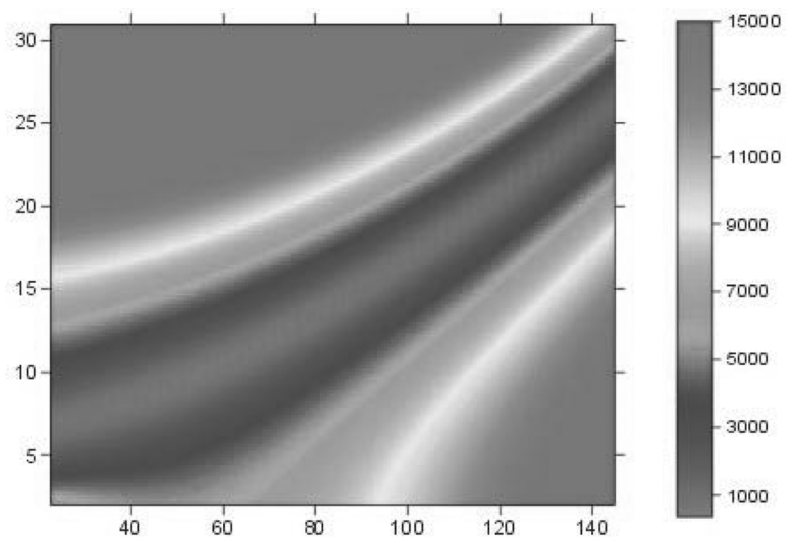

(a)

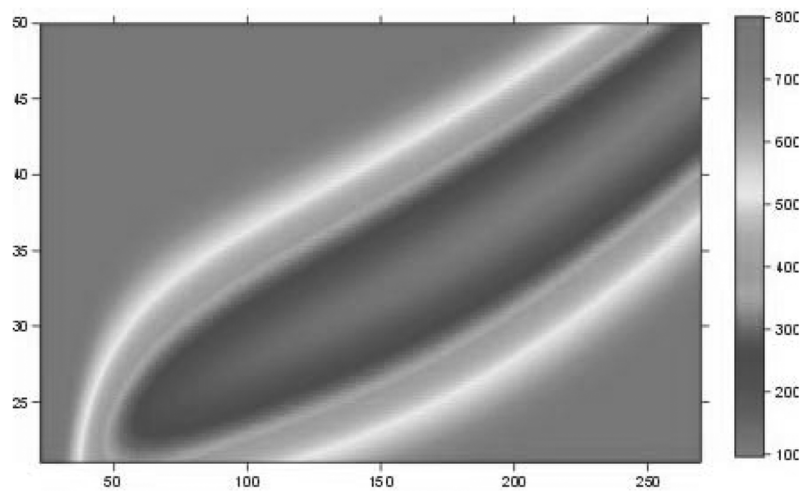

(b)

Fig. 3. The absolute error of traveltime with respect to frequency and damping factor for homogeneous models: (a) $\mathrm{v}=2$ $\mathrm{km} / \mathrm{s}$ and (b) $\mathrm{v}=8 \mathrm{~km} / \mathrm{s}$. The grid interval is $8 \mathrm{~m}$. The number of grids is $1151 \times 376$.

placed at the $600^{\text {th }}$ grid at the surface. Then, we compute the traveltime error for this homogeneous model as a function of the frequency and damping factor. Fig. 3a shows the total absolute error between the computed traveltime and the analytic traveltime. In Fig. 3a, the sum of the traveltime errors is $387.56 \mathrm{~s}$ and the corresponding frequency and damping factor as $10 \mathrm{~Hz}$ and 55, respectively. Thus, the average error for this model becomes $0.0008955 \mathrm{~s}$. The traveltime computed using the optimum complex frequency is shown in Fig. 4a. Additionally, the error between the numerical traveltime computed with the optimum complex frequency and the analytic traveltime is shown in Fig. 4b. From Fig. 4b, we note that the computed trav-

Table 1. Comparison of the error of our method with that of the method used by Shin et al. (2003a). The average error of our method is much smaller than that of Shin et al. (2003a), although the size of our model is 8 times larger than that used in Shin et al. (2003a).

\begin{tabular}{cccccc}
\hline & Grid size & Velocity & Number of grids & Maximum error & Average error \\
\hline Shins method & $5 \mathrm{~m}$ & $1.5 \mathrm{~km} / \mathrm{s}$ & $401 * 201$ & $0.0055 \mathrm{~s}$ & $0.0025 \mathrm{~s}$ \\
\hline Our method & $8 \mathrm{~m}$ & $2 \mathrm{~km} / \mathrm{s}$ & $1151 * 376$ & $0.004 \mathrm{~s}$ & $0.00089 \mathrm{~s}$ \\
\hline
\end{tabular}




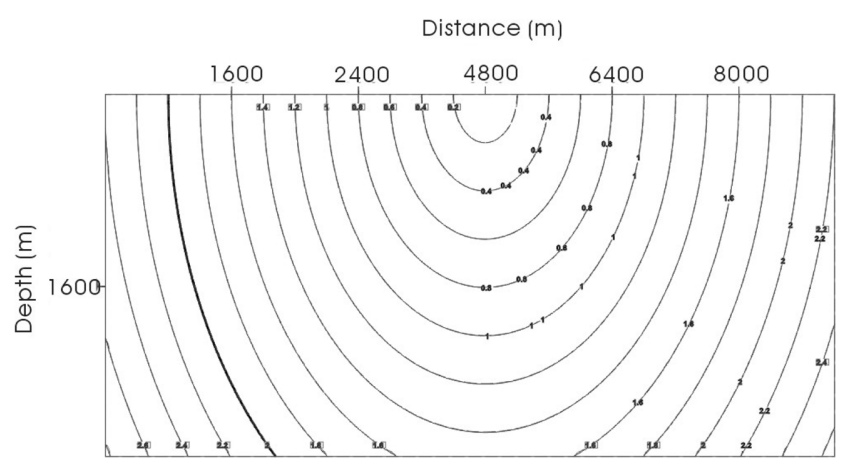

(a)

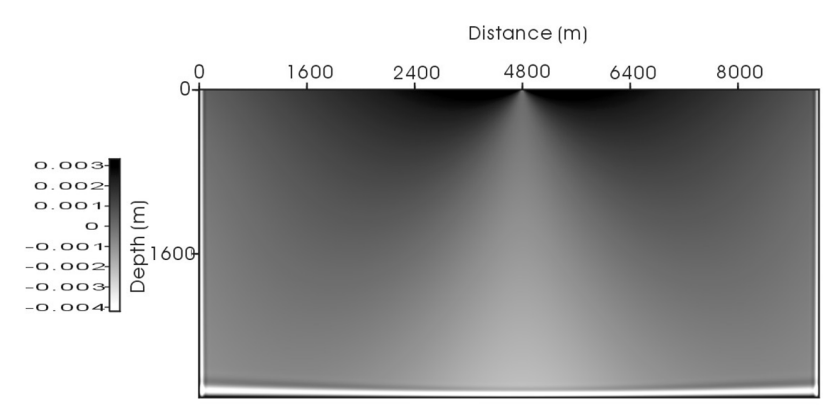

(b)

Fig. 4. (a) Analytic (solid line) and numerical traveltimes computed by using the optimum frequency and damping factor (dotted line) for the homogeneous model whose velocity is $2 \mathrm{~km} / \mathrm{s}$; (b) The corresponding errors between the analytic traveltimes and the numerical traveltimes.

eltime are compatible with the analytic traveltime. In Fig. $4 \mathrm{~b}$, all of the errors are less than $0.004 \mathrm{~s}$, and the average error is $0.0008955 \mathrm{~s}$. In Table 1, we compare the error of our algorithm with that of the basic algorithm suggested by Shin et al. (2003a). From Table 1, we can note that the average error of our algorithm is much smaller than that of the basic algorithm of Shin et al. (2003a), although the size of our model is eight times larger than the model used in Shin et al. (2003a).

\section{Improvement on the stability}

Using parameter analysis, we can get the optimum combination of frequency and damping factor for a homogeneous media with a velocity of $2 \mathrm{~km} / \mathrm{s}$. In order to estimate how the optimum frequency and damping factor

Table 2. Optimum complex frequency for different velocities in homogeneous media.

\begin{tabular}{cccc}
\hline Velocity & Grid size & $\begin{array}{c}\text { Optimum } \\
\text { Frequency }\end{array}$ & $\begin{array}{c}\text { Optimum } \\
\text { Damping Factor }\end{array}$ \\
\hline $2 \mathrm{~km} / \mathrm{s}$ & $8 \mathrm{~m}$ & $10 \mathrm{~Hz}$ & 55 \\
\hline $4 \mathrm{~km} / \mathrm{s}$ & $8 \mathrm{~m}$ & $20 \mathrm{~Hz}$ & 110 \\
\hline $8 \mathrm{~km} / \mathrm{s}$ & $8 \mathrm{~m}$ & $40 \mathrm{~Hz}$ & 220 \\
\hline
\end{tabular}

varies with the velocity, we apply the parameter analysis to another homogenous model with a different velocity. Fig. $3 \mathrm{~b}$ is the result of parameter analysis for one model with a constant velocity of $8 \mathrm{~km} / \mathrm{s}$. From Fig. 3b, we can know that the optimum frequency and damping factor is $40 \mathrm{~Hz}$ and 220 , respectively, when the velocity is $8 \mathrm{~km} / \mathrm{s}$. The comparison of the optimum frequency and damping factor for the two different models is shown in Table 2. From Table 2, we note that there is a linear relationship between velocity and optimum frequency or optimum damping factor. Therefore, we obtain the following empirical formula for our numerical method

$$
\omega^{*}=\frac{2 \pi V}{G_{f} \Delta}+i \frac{2 \pi V}{G_{\alpha} \Delta}
$$

where the $G_{f}=25, G_{\alpha}=25$, and $\Delta$ is the grid size.

Equation (15) is accurate for homogeneous media. For heterogeneous media, we usually replace the velocity term in equation (15) with a minimum velocity and average velocity as

$$
\omega^{*}=\frac{2 \pi V_{\min }}{G_{f} \Delta}+i \frac{2 \pi V_{a v g}}{G_{\alpha} \Delta}
$$

where $V_{\min }$ and $V_{\text {avg }}$ is the minimum velocity and the aver-

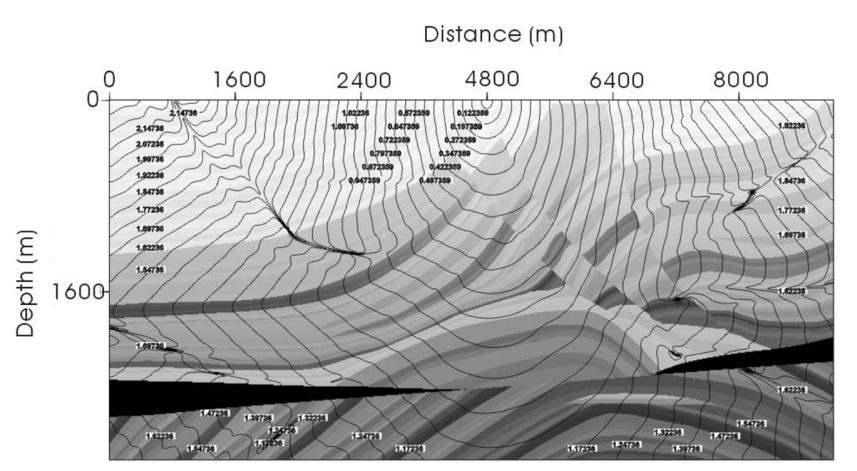

(a)

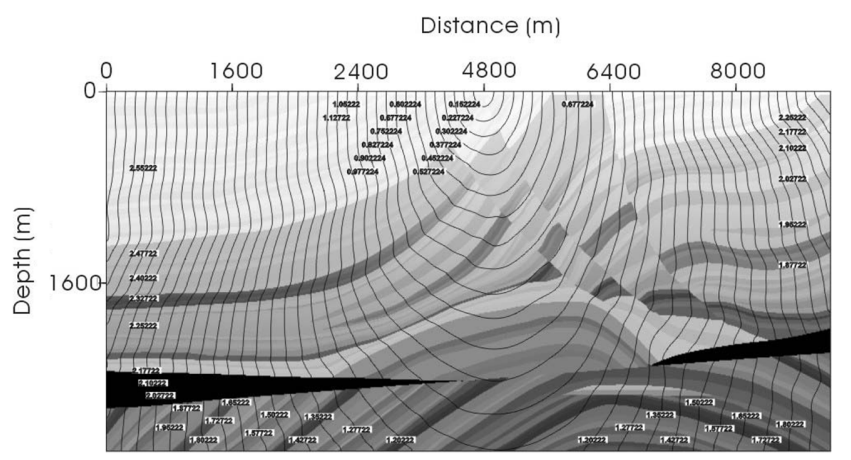

(b)

Fig. 5. Marmousi model overlaid by traveltime contour calculated by using (a) the method of Shin et al. (2003a) and (b) the adaptive frequency and the adaptive damping factor. 
age velocity, respectively, in the inhomogeneous model. The traveltime for the Marmousi model computed with equation (16) is shown in Fig. 5a. In Fig. 5a, we observe that the traveltime contour is obviously distorted. Shin et al. (2003a) speculated that the distorted contours of traveltimes resulting from multiple events with nearly the same amplitude as or larger amplitude than that of the firstarrival event.

To overcome the problem, we propose the following adaptive optimum frequency and the damping factor for heterogeneous media

$$
\omega^{*}(i, j)=\frac{2 \pi V(i, j)}{G_{f} \Delta}+i \frac{2 \pi V(i, j)}{G_{\alpha} \Delta}
$$

where the $G_{f}=25, G_{\alpha}=28.54, \Delta$ is the grid size, $i$ and $j$ is the vertical coordinate and horizontal coordinate, respectively. From equation (17), we can note that the parameter $G$ changes according to frequency and damping factor. The traveltime for the Marmousi model, computed using equation (17), is shown in Fig. 5b. From Fig. 5b, we can see that the distortion observed in Fig. 5a disappears.

\section{COMPARISON WITH OTHER TRAVELTIMES}

Having successfully demonstrated that our algorithm has both higher accuracy and better stability, we proceeded to compare the travel times obtained by our method with the most-energetic traveltime obtained with the method used by Shin et al. (2003b) and the traveltime obtained by the (Lim et al., 2002). In Fig. 6a, we display the traveltime contours computed by our method and the most-energetic traveltime. In Fig. 6a, we note that the traveltime computed by our method shows good agreement with the mostenergetic traveltime. From the traveltime contour far away from source in Fig. 6a, we note that the traveltime computed by our algorithm is comparable to the transmitted waves rather than the first arrival. We also compared our traveltime with the traveltime generated by the SRT in Fig. 6b. The traveltime obtained by the SRT approximately describes the direct wave. From Fig. 6b, we note that our traveltime contour has good agreement with that of the SRT. To verify further that our traveltime is very close to the transmitted wave, rather than the head wave, we devised a simple model with three layers. The velocity of each layer from the top to the lower is 1500, 2000 and $2800 \mathrm{~m} / \mathrm{s}$. In Fig. 7a, we overlap the resulting traveltime contours on the velocity model. In Fig. 7a, we observe that there is head wave in the zone far away from the source, and the traveltime contour in the zone far away from the source obviously corresponds to the transmitted wave. In Figs. 7b and 7c, we also compute the transmittedwave traveltime using a one-way wave equation and the first-arrival traveltime using the finite-difference method of Vidale (1988).

Next, we check whether or not the traveltime obtained by

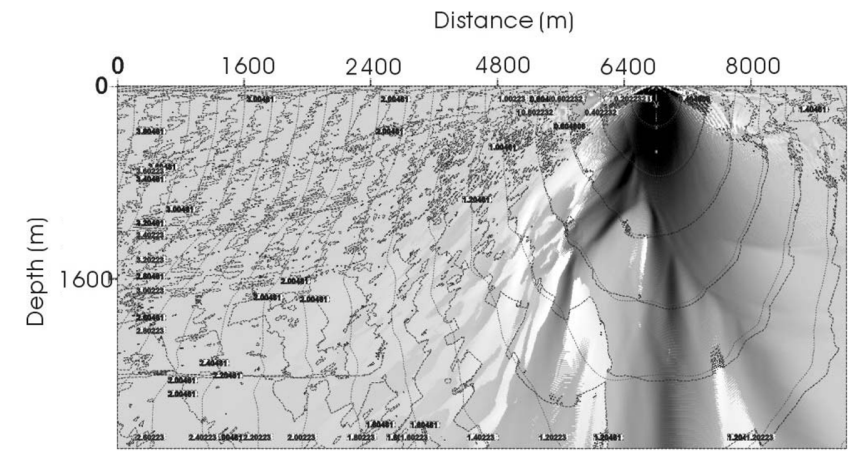

(a)

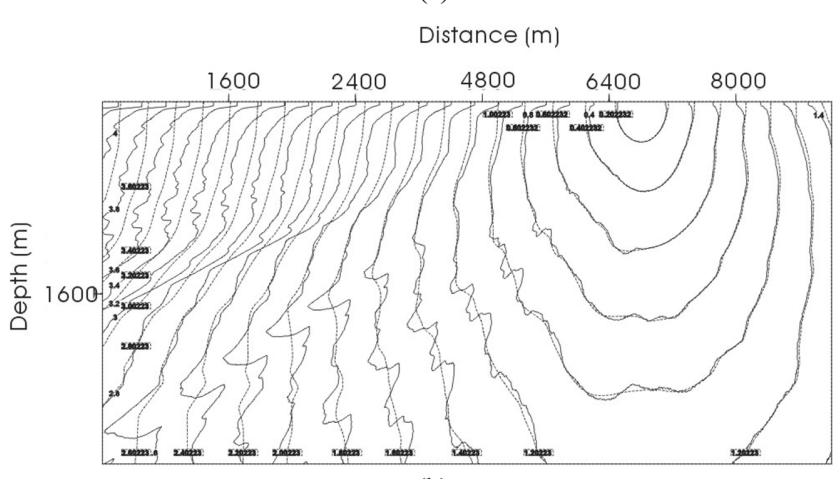

(b)

Fig. 6. (a) Traveltime contours calculated by our method (dotted line) and the most energetic traveltime contour (solid line) obtained with the method used by Shin et al. (2003b) for the Marmousi model, superimposed on the amplitude image of the most energetic. The velocities change from $1500 \mathrm{~m} / \mathrm{s}$ at the top of the model to 5500 $\mathrm{m} / \mathrm{s}$ at the bottom of the model; and (b) the comparison between our traveltime (dotted line) and the SRT traveltime (solid line) for the Marmousi model.

our method yielded good migration images. Figs. 8a and $8 \mathrm{~b}$ show the prestack Kirchhoff migration images using only the traveltime computed with our method and the most-energetic traveltime obtained by using the method of Shin et al. (2003b). From Fig. 8a, we note that the migration image obtained by our traveltime is better than the result computed by the most-energetic traveltime. We also added the most-energetic amplitude to the corresponding traveltime to get the Kirchhoff migration images, as shown in Figs. 9a and 9b. In Fig. 9, we note that the images computed by our traveltime give better resolved faults and reservoir and that the results are compatible with the images computed by the most-energetic traveltime and amplitude.

\section{CONCLUSIONS}

By calculating traveltime error as the function of frequency and damping factor for different velocities when the grid size is fixed, we obtain the optimum frequency and the optimum damping factor for different velocities. We 


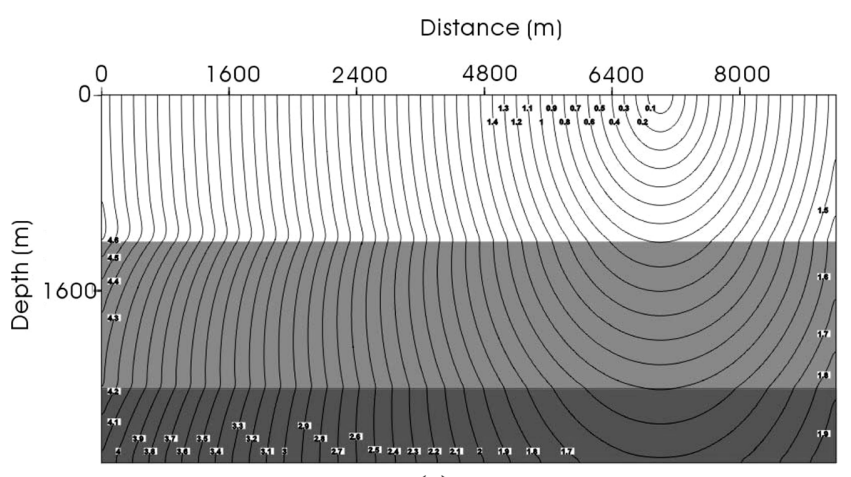

(a)

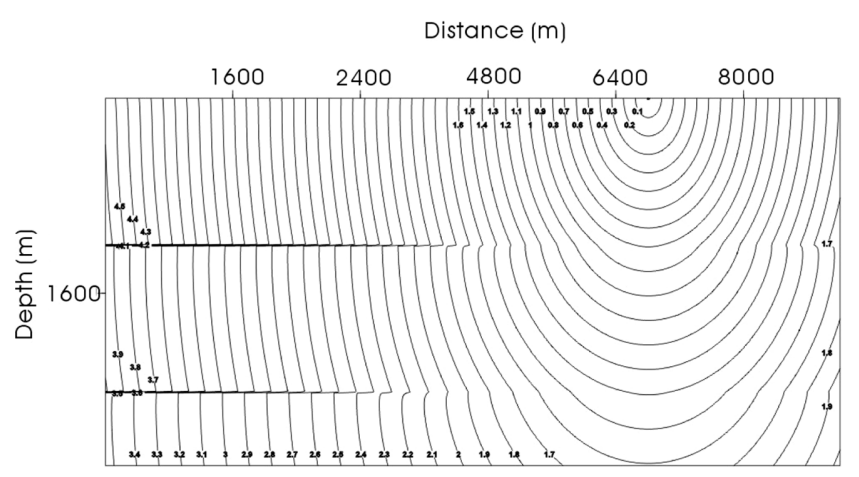

(b)

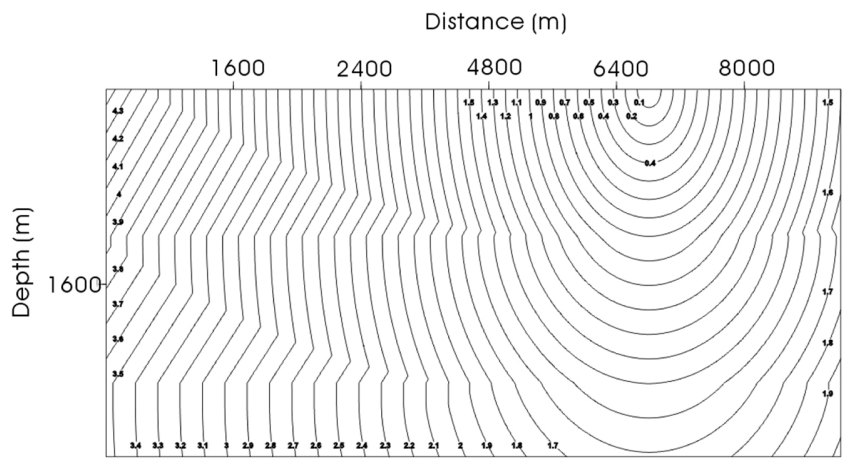

(c)

Fig. 7. (a) Traveltime computed by our method for a three-layer model, (b) the transmitted-wave traveltime computed using a one-way wave equation. (c) the first-arrival traveltime computed by using Vidales method (1988). The velocity of each layer from the top to the lower is 1500 , 2000 and $2800 \mathrm{~m} / \mathrm{s}$, respectively

then derive an accurate empirical formula for our numerical algorithm. The accuracy of traveltime is much improved by using the optimum frequency and the optimum damping factor derived from the parameter analysis. We applied the adaptive frequency and the adaptive damping factor to eliminate the distorted traveltime contour for heterogeneous media. Compared with the most-energetic traveltime and the SRT traveltime, our algorithm calculates the traveltime that is comparable to the directly transmitted wave rather than the head wave.

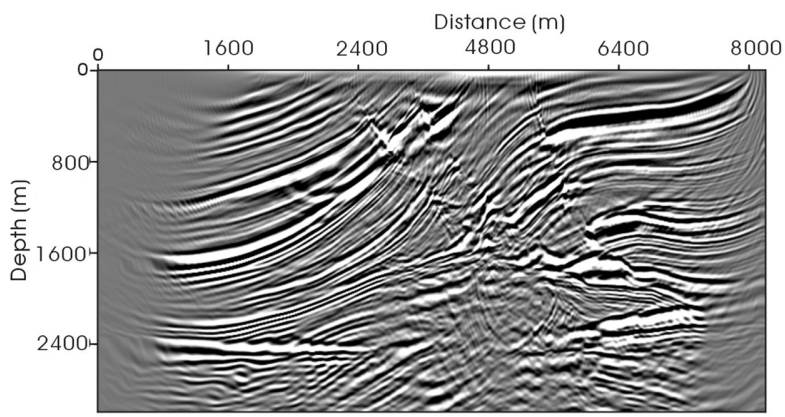

(a)

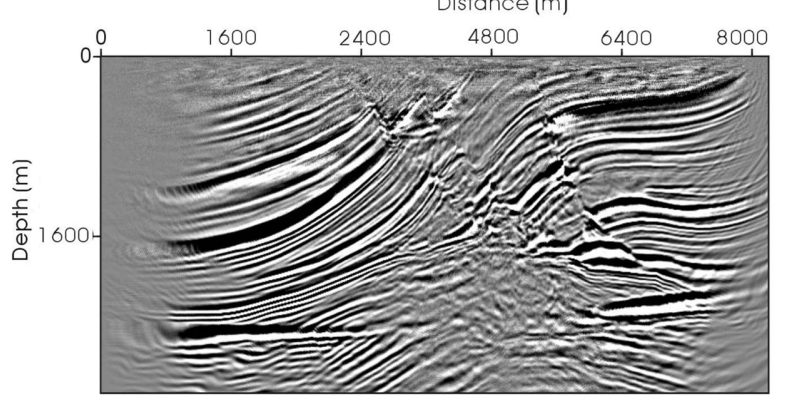

(b)

Fig. 8. Prestack Kirchhoff migration images for the Marmousi model using (a) traveltime obtained by our algorithm and (b) the most-energetic traveltime obtained by Shin et al. (2003b)

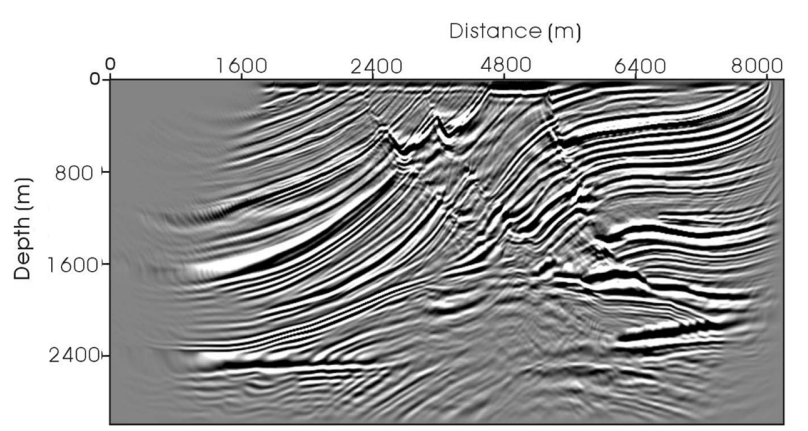

(a)

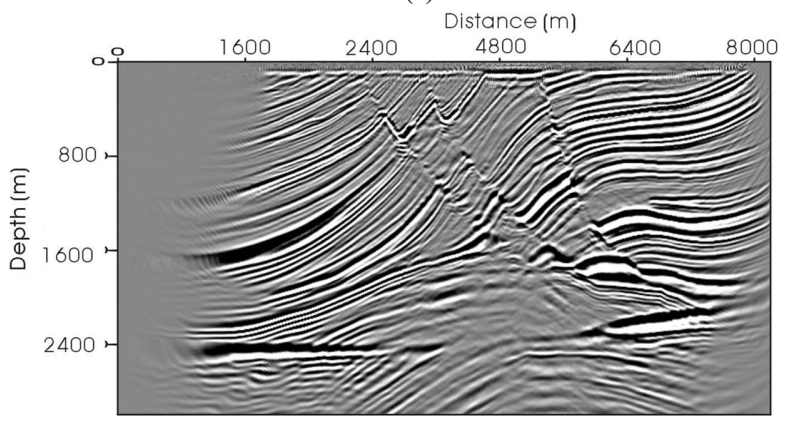

(b)

Fig. 9. Prestack Kirchhoff migration images generated by using (a) the traveltime obtained by our algorithm and (b) the most energetic traveltime obtained by Shin et al. (2003b) for the Marmousi model. For two migration images, we use the same amplitudes. 


\section{ACKNOWLEGEMENTS}

This study is sponsored by the Chinese State Natural Science Foundation (49825108), the Chinese Academy of Sciences (KZCX2-109 and KZ951-B1-407-02), and the Korea Foundation for Advanced Studies. This work was also financially supported by the National Laboratory Project of the Ministry of Science and Technology and the Brain Korea 21 project of the Ministry of Education.

\section{REFERENCES}

Afnimar and Koketsu, K., 2000, Finite difference traveltime calculation for head waves traveling along an irregular interface: Geophysical Journal International, Vol. 143, p. 729-734

Alkhalifah, T. and Fomel, S., 2001, Implementing the fast marching eikonal solver: spherical versus Cartesian coordinates: Geophysical Prospecting, Vol. 49, p. 165-178.

Cao, S. and Greenhalgh, S. A., 1993, Calculation of the seismic first-break time field and its ray path distribution using a minimum traveltime tree algorithm: Geophysical Journal International, Vol. 114, p. 593-600.

Cheng, N. and House, L., 1996, Minimum traveltime calculation in 3-D graph theory: Geophysics, Vol. 61, p. 18951898.

Fischer, R. and Lees, J. M., 1993, Shortest path ray tracing with sparse graphs: Geophysics, Vol. 58, p. 987-996.
Kim, S., 2002, 3-D eikonal solvers: First-arrival traveltimes: Geophysics, Vol. 67, p. 1225-1231.

Lim, H. Y., Min, D. -J., Shin C., and Yang, D., 2002, Prestack depth migration using Straight Ray Technique (SRT): Journal of Seismic Exploration, Vol. 11, p. 271281.

Mo, L. W. and Harris, J. M., 2002, Finite-difference calculation of direct-arrival traveltimes using the eikonal equation: Geophysics, Vol. 67, p. 1270-1274.

Nichols, D. E., 1996, Maximum energy traveltimes calculated in the seismic frequency band: Geophysics, Vol. 61, p. 253263.

Shin, C., Ko, S., Kim, W., Min, D. J., Yang, D. and Marfurt K. J., 2003a, Traveltime calculations from frequencydomain downward-continuation algorithms. Geophysics, Vol. 68 p. $1380-1388$

Shin, C., Ko, S. W., Marfurt, K. J. and Yang, D., 2003b, Wave equation calculation of most energetic traveltimes and amplitudes for Kirchhoff prestack migration: Geophysics, Vol. 68, p. 2040-2042.

Shin, C., Min, D. J., Marfurt, K. J., Lim, H.Y., Yang D., Cha, Y., Ko, S., Ha, T. and Hong, S., 2002, Traveltime and amplitude calculations using the damped wave solution: Geophysics, Vol. 67, p. 1637-1647.

Vidale, J., 1988, Finite-difference calculation of travel times: Bulletin of the Seismological Society of America, Vol. 78, p. 2062-2076. 
Zhang Zhongjie received Ph.D (1991) in Geophysics from Jilin University. He worked in the Standford University as a visiting scholar and visiting professor in 1996 and 2000, respectively. Presently, he is a research professor in the Institute of Geology \& Geophysics, Chinese Academy of Sciences (CAS). His current research interest includes seismic anisotropic modeling, tomography, crust/mantle structure and geodynamics.(E-mail: zhangzj@ mail.iggcas.ac.cn)

Qin Yilong received a BS (1997), an MS (2000) in exploration geophysics from Daqing Petroleum Institute, and Ph.D (2003) in seismology from the Institute of Geology \& Geophysics, Chinese Academy of Sciences (CAS). Currently, he is a research assistant in the Institute of Geology \& Geophysics, CAS, and his current research interest includes traveltime calculation in anisotropic media, Kirchhoff migration and traveltime tomography.(E-mail: yilongqin@mail.igcas.ac.kr)

Chen Yun received a BS (1997) in Geophysics from Jilin University and MS (2004) in exploration geophysics from China University of Geosciences, Beijing. From 1997 to present, he works in the Institute of Geology \& Geophysics, Chinese Academy of Sciences (CAS). His current research interest includes seismic tomography, wavefield analysis and seismic anisotropy.(E-mail: yunchen@mail.iggcas.ac.cn)

Changsoo Shin received a $\mathrm{PhD}$ (1988) in geophysics from the University of Tulsa. After working at the Korea Institute of Geology, Mining, and Materials from 1990 to 1996, he

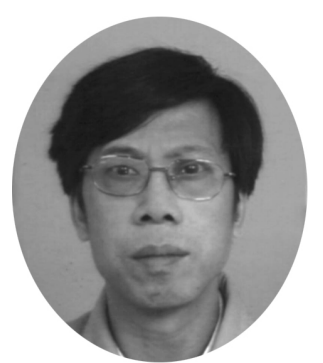

Zhang Zhongjie

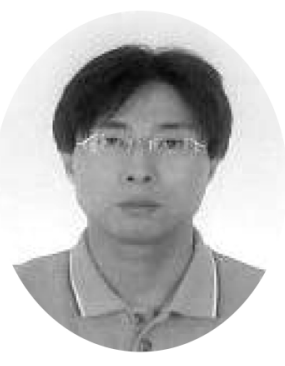

Seungwon Ko

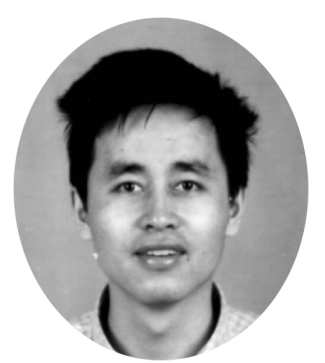

Qin Yilong

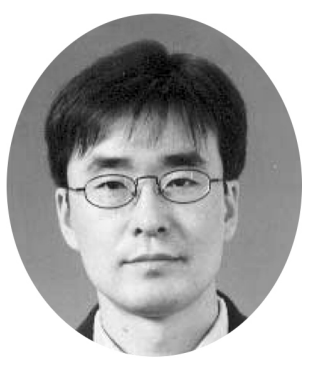

Young Tak Seo

became an associate professor of geophysics at Seoul National University. He has led the national research laboratory of seismic inversion and imaging of Seoul National University since June 2000. His interests are the numerical modeling of wave propagation, full waveform inversion, and geophysical imaging. (E-mail: css@model.snu.ac.kr)

Seungwon Ko received his BS(1994), MS(1996), Ph.D(2001) from Hanyang University. He was a visiting scholar in AGL (Allied Geophysical Laboratory) in Houston after his Ph.D and is currently working in technical department of KNOC(Korea National Oil Corporation). His main research area is seismic data processing and especially, scalar and vector PSDM (Prestack Depth Migration) and seismic inversion. (E-mail: ko@gpl.snu.ac.kr)

Young Tak Seo is a student in Ph.D course in Civil, Urban and Geosystem Engineering at Seoul National University. He holds BS degree in Mineral and Resources Engineering from Semyung University and MS degree in Geophysical Prospecting from Seoul National University.(E-mail: geoneer@empal. com)

Han Uk received a BS(1970), an MS(1974) in Astronomy from Seoul National University, and MS(1979)/Ph.D(1984) in Thermal Processes and Tectonophysics from the University of Utah, SLC, USA. Currently, he is a professor in the Dept. of Environmental Sci., Korea Military Academy.(E-mail: jigugong @ chollian.net)

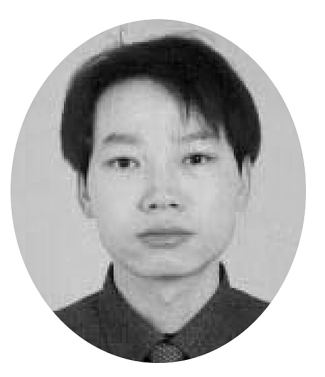

Chen Yun

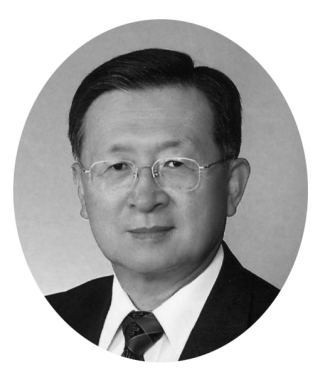

Han Uk

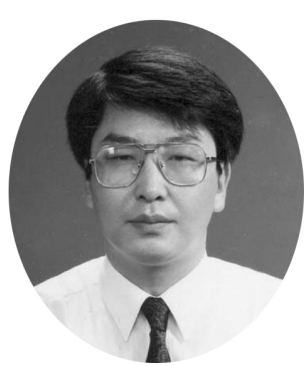

Changsoo Shin 